\title{
The Differential Impact of SRC Expression on the Prognosis of Patients with Head and Neck Squamous Cell Carcinoma
}

\author{
Francisco Hermida-Prado ${ }^{1,2,+}+\mathbb{D}$, Rocío Granda-Díaz ${ }^{1,2,+}$, Nagore del-Río-Ibisate ${ }^{1,2,+}$, \\ M. Ángeles Villaronga ${ }^{1,2}$, Eva Allonca ${ }^{1,2}{ }^{(D}$, Irati Garmendia ${ }^{3}\left(\mathbb{D}\right.$, Luis M. Montuenga ${ }^{2,3}(\mathbb{D})$, \\ René Rodríguez ${ }^{1,2}$, Aitana Vallina ${ }^{4}$, César Alvarez-Marcos ${ }^{1,2}$, Juan P. Rodrigo ${ }^{1,2, *(D)}$ \\ and Juana M. García-Pedrero ${ }^{1,2, *(D)}$
}

1 Department of Otolaryngology, Hospital Universitario Central de Asturias and Instituto de Investigación Sanitaria del Principado de Asturias (ISPA), Instituto Universitario de Oncología del Principado de Asturias, University of Oviedo, 33011 Oviedo, Spain; franjhermida@gmail.com (F.H.-P.); rocigd281@gmail.com (R.G.-D.); nagoredelrio@gmail.com (N.d.-R.-I.); angelesvillaronga@gmail.com (M.Á.V.); ynkc1@hotmail.com (E.A.); renerg.finba@gmail.com (R.R.); caalvarez@uniovi.es (C.A.-M.)

2 Ciber de Cáncer, CIBERONC, Instituto de Salud Carlos III, Av. Monforte de Lemos, 3-5, 28029 Madrid, Spain; lmontuenga@unav.es

3 Program in Solid Tumors, Center for Applied Medical Research (CIMA), Department of Pathology, Anatomy and Physiology, University of Navarra, and Navarra's Health Research Institute (IDISNA), 31008 Pamplona, Spain; igarmendia@alumni.unav.es

4 Department of Pathology, Hospital Universitario Central de Asturias and Instituto de Investigación Sanitaria del Principado de Asturias (ISPA), Instituto Universitario de Oncología del Principado de Asturias, University of Oviedo, 33011 Oviedo, Spain; alaicla@hotmail.es

* Correspondence: jprodrigo@uniovi.es (J.P.R.); juanagp.finba@gmail.com (J.M.G.-P.)

+ These authors contributed equally to this work.

Received: 3 October 2019; Accepted: 23 October 2019; Published: 25 October 2019

\begin{abstract}
Aberrant SRC expression and activation is frequently detected in multiple cancers, and hence, targeting SRC has emerged as a promising therapeutic strategy. Different SRC inhibitors have demonstrated potent anti-tumor activity in preclinical models, although they largely lack clinical efficacy as monotherapy in late-stage solid tumors, including head and neck squamous cell carcinomas (HNSCC). Adequate selection and stratification of patients who may respond to and benefit from anti-SRC therapies is therefore needed to guide clinical trials and treatment efficacy. This study investigates the prognostic significance of active SRC expression in a homogeneous cohort of 122 human papillomavirus (HPV)-negative, surgically treated HNSCC patients. Immunohistochemical evaluation of the active form of SRC by means of anti-SRC Clone 28 monoclonal antibody was specifically performed and subsequently correlated with clinical data. The expression of p-SRC (Tyr419), total SRC, and downstream SRC effectors was also analyzed. Our results uncovered striking differences in the prognostic relevance of SRC expression in HNSCC patients depending on the tumor site. Active SRC expression was found to significantly associate with advanced disease stages, presence of lymph node metastasis, and tumor recurrences in patients with laryngeal tumors, but not in the pharyngeal subgroup. Multivariate Cox analysis further revealed active SRC expression as an independent predictor of cancer-specific mortality in patients with laryngeal carcinomas. Concordantly, expression of p-SRC (Tyr419) and the SRC substrates focal adhesion kinase (FAK) and the Arf GTPase-activating protein ASAP1 also showed specific associations with poor prognosis in the larynx. These findings could have important implications in ongoing Src family kinase (SFK)-based clinical trials, as these new criteria could help to improve patient selection and develop biomarker-stratified trials.
\end{abstract}


Keywords: head and neck squamous cell carcinoma; immunohistochemistry; SRC; prognosis; larynx; pharynx

\section{Introduction}

Head and neck squamous cell carcinoma (HNSCC) represents the sixth most common cancer worldwide. The latest advancements in cancer diagnosis and treatment have led to only modest improvements of survival rates for HNSCC patients [1]. It has become clear by omics studies that HNSCC is a highly complex and heterogeneous disease [2], involving multiple different genetic and molecular alterations that ultimately hamper our ability to accurately predict aggressive tumor behavior. The identification of novel markers capable of distinguishing the biological behavior of tumors could certainly contribute to improve predictability beyond the current clinicopathological markers.

SRC belongs to the highly conserved family of non-receptor protein tyrosine kinases known as the Src family kinases (SFKs) that includes SRC, BLK, FGR, FRK, FYN, HCK, LCK, LYN, YES, and YRK $[3,4]$. Each family member has shown a different expression pattern and tissue distribution. $\mathrm{SRC}$ is one of the oldest and most investigated proto-oncogenes. Mounting evidence demonstrates that SRC plays a pivotal role at different stages of tumorigenesis [4-6], thereby modulating multiple oncogenic signaling pathways and biological processes essential for the malignant phenotype, such as proliferation, cell adhesion, motility, invasion, and angiogenesis [5-9]. It has been reported that SRC interacts with multiple receptor tyrosine kinases (RTKs), including, among others, epidermal growth factor receptors (EGFR and HER2), insulin growth factor receptor IGF-1R, fibroblast growth factor receptor, platelet-derived growth factor receptor, hepatocyte growth factor receptor c-MET, and c-Kit [10]. SRC is a critical player in the regulation of cellular adhesion mediated by integrins, epithelial-to-mesenchymal transition via E-cadherin suppression, and focal adhesions by activation of focal adhesion kinase (FAK) [11-13]. In addition, SRC regulates the EGF-dependent actin cytoskeleton reorganization via p190 phosphorylation [14]. Cortactin (CTTN) is another important SRC substrate that participates in actin remodeling and invadopodium formation. It has been described that SRC-mediated phosphorylation of CTTN modulates its interaction with FAK at focal adhesions, thereby promoting cell motility. On this basis, it has been suggested that CTTN may act as a bridge between actin filaments and focal adhesions [15]. The Arf GTPase-activating protein (GAP) ASAP1 (also known as AMAP1 or DDEF1) has also emerged as a key SRC substrate that regulates cytoskeleton dynamics and invadopodium formation by binding to both CTTN and paxillin, thereby playing a critical role in promoting invasion and metastasis [16,17].

Elevated SRC expression and activity has been frequently detected in multiple cancers, including breast, ovarian, colon, pancreatic, gastric, hepatocellular, lung, bladder esophageal, and HNSCC [9,18-21]. These observations led to rapid development of SRC inhibitors, such as dasatinib (BMS354825), saracatinib (AZD0530), and bosutinib (SKI-606), which have been actively tested in preclinical settings and also in clinical trials for multiple cancers [4,21-32]. However, disappointingly, SRC inhibitors did not show any significant activity as monotherapeutic agents in the treatment of patients with advanced-stage solid tumors [21,23-25,29,30], including HNSCC [33,34].

Human SRC protein is a tyrosine kinase structurally composed of four Src-homology (SH1-4) domains that mediate the regulation of SRC kinase activity and also protein interaction with a large number of substrates to form intracellular signaling complexes [5,7,10]. In addition, full activation of SRC is tightly regulated and dependent on the phosphorylation of residue Tyr419, while Tyr530 phosphorylation is involved in its inactivation. The generation of a monoclonal antibody (Clone 28) specific for the C-terminal regulatory domain of human c-Src that selectively recognizes the active form of SRC (not phosphorylated at Tyr530) was previously reported [35]. Hence, this antibody emerged as a useful tool to specifically detect the active form of SRC protein in both tissues and cells. 
The present study is the first to investigate the expression pattern and clinical significance of active SRC in a homogeneous cohort of 122 human papillomavirus (HPV)-negative HNSCC patients treated by surgery at the same institution. Our results reveal clear differences in the prognostic relevance of SRC expression in HNSCC patients depending on the tumor site. Thus, aberrant SRC expression/activation was found to significantly associate with advanced disease stages, presence of lymph node metastasis, and tumor recurrences in patients with laryngeal tumors, but not in the pharyngeal subgroup. Moreover, active SRC expression now emerges as an independent predictor of cancer-specific mortality in laryngeal cancer. Similarly, the expression of p-SRC (Tyr419) and various downstream SRC effectors such as FAK and ASAP1 significantly and consistently correlated with active SRC expression and also specifically predicted poor prognosis in the larynx. These novel findings could be useful to improve the selection of patients who can benefit from treatment with anti-SRC therapies. Accordingly, biomarker-based patient stratification could help to refine eligibility criteria and guide clinical trials, and to ultimately improve treatment efficacy and long-term clinical outcomes.

\section{Materials and Methods}

\subsection{Patients and Tissue Specimens}

Surgical tissue specimens were collected from 122 patients with laryngeal or hypopharyngeal squamous cell carcinoma surgically treated at the Hospital Universitario Central de Asturias (HUCA) between 1996 and 2005, in accordance with approved Institutional Review Board guidelines. Experimental procedures were performed in accordance with the Declaration of Helsinki. Written informed consent was obtained from all patients. Formalin-fixed paraffin-embedded (FFPE) tissue samples and data from donors were provided by the Principado de Asturias BioBank (PT17/0015/0023), integrated in the Spanish National Biobanks Network, and histological diagnosis was confirmed by an experienced pathologist. Samples were processed following standard operating procedures with the appropriate approval of the Ethical and Scientific Committees of the HUCA and the Regional CEIC from Principado de Asturias for the project PI16/00280 (approval number: 70/16; date: 5 May 2016).

All patients had a single primary tumor and microscopically clear surgical margins. Patients did not receive any treatment prior to surgery. Only five patients were women. The mean age was 60 years (range 38 to 86 years). Of the 122 patients, 119 were habitual tobacco smokers, 71 moderate (1-50 pack-years) and 48 heavy (>50 pack-years), and 107 were habitual alcohol drinkers; further, $66(54 \%)$ of the 122 patients received postoperative radiotherapy. The characteristics of the patients studied and the clinicopathologic features of their tumors are summarized in Table 1 and Supplementary Table S1. The stage of disease was determined after the surgical resection of the tumor according to the TNM system of the International Union against Cancer (7th Edition). The histological grade was determined according to the degree of differentiation of the tumor (Broders' classification). The HPV status was available for all the patients, determined as previously reported [36].

\subsection{Immunohistochemical Analysis of Patient Samples}

Three morphologically representative areas were selected from each individual tumor block to construct five tissue microarray (TMA) blocks, as described previously [36]. Each TMA also contained three cores of normal epithelium as an internal control. TMA blocks were cut into $3 \mu \mathrm{m}$ sections and dried on Flex IHC microscope slides (Dako, Glostrup, Denmark). TMA sections were deparaffinized, and antigen retrieval was carried out by using Envision Flex Target Retrieval solution (Dako, Glostrup, Denmark), high $\mathrm{pH}$ or low $\mathrm{pH}$ (for active SRC) or proteinase K (for ASAP1). Staining was performed at room temperature on an automatic staining workstation (Dako Autostainer Plus) with SRC (active) monoclonal antibody Clone 28 (Thermo Fisher Scientific \#AHO0051) at 1:300 dilution, phospho-SRC (Y419) antibody (Invitrogen \# 44-660G) at 1:80 dilution, total SRC (Santa Cruz Biotechnology \# sc-8056) at 1:100, anti-ASAP1 antibody (abcam \# 11011) at 1:750, mouse anti-cortactin monoclonal antibody Clone 30 (BD Biosciences Pharmingen \# 610049) at 1:200 dilution, or mouse anti-FAK monoclonal 
antibody Clone 4.47 (Merck Millipore \# 05-537) at 1:250 dilution using the Dako EnVision Flex+ Visualization System (Dako Autostainer, Denmark). Counterstaining with hematoxylin was the final step.

Table 1. Associations between the expression of active SRC and clinicopathological parameters, recurrence, and disease outcome.

\begin{tabular}{|c|c|c|c|}
\hline Characteristic & No. & Active SRC Expression (\%) & $P$ \\
\hline \multicolumn{4}{|l|}{ pT classification } \\
\hline $\mathrm{T} 1, \mathrm{~T} 2$ & 32 & $21(66)$ & \multirow[b]{2}{*}{$0.145^{\dagger}$} \\
\hline $\mathrm{T} 3, \mathrm{~T} 4$ & 84 & $67(79)$ & \\
\hline \multicolumn{4}{|l|}{$\mathrm{pN}$ classification } \\
\hline No & 39 & $24(62)$ & \multirow[b]{2}{*}{$0.020^{\dagger}$} \\
\hline N1-3 & 77 & $64(83)$ & \\
\hline \multicolumn{4}{|l|}{ Disease stage } \\
\hline I, II & 15 & $8(53)$ & \multirow{2}{*}{$0.048^{\dagger}$} \\
\hline III, IV & 101 & $80(79)$ & \\
\hline \multicolumn{4}{|l|}{ Pathological grade } \\
\hline Well differentiated & 33 & $22(67)$ & \multirow{3}{*}{$0.286^{\#}$} \\
\hline Moderately differentiated & 53 & $41(77)$ & \\
\hline Poorly differentiated & 30 & $25(83)$ & \\
\hline \multicolumn{4}{|l|}{ Site } \\
\hline Pharynx & 58 & $48(83)$ & \multirow{2}{*}{$0.128^{\dagger}$} \\
\hline Larynx & 58 & $40(69)$ & \\
\hline \multicolumn{4}{|l|}{ Tumor Recurrence $\ddagger$} \\
\hline No & 26 & $14(54)$ & \multirow{2}{*}{$0.002^{\dagger}$} \\
\hline Yes & 68 & $58(85)$ & \\
\hline \multicolumn{4}{|l|}{ Disease status } \\
\hline Alive without disease & 30 & $16(53)$ & \multirow{3}{*}{$0.001^{\dagger}$} \\
\hline Dead of index cancer & 64 & $56(88)$ & \\
\hline Died of other causes & 22 & $16(73)$ & \\
\hline Total Cases & 116 & $88(76)$ & \\
\hline
\end{tabular}

\footnotetext{
${ }^{\#}$ Chi-square and ${ }^{\dagger}$ Fisher's exact tests. ${ }^{\ddagger}$ The 22 patients who died of cancer-unrelated causes were excluded from the recurrence analysis.
}

Immunostaining was scored blinded to clinical data by two independent observers. Since SRC, ASAP1, CTTN, and FAK staining showed a homogeneous distribution, a semiquantitative scoring system was applied, and staining intensity was scored as negative ( 0$)$, weakly $(1+)$, moderately $(2+)$, or strongly positive (3+). Scores of $\geq 2$ were considered as positive expression. $p$-SRC (Tyr419) expression was detected in both the nucleus and cytoplasm of tumor cells and scored as negative (0) versus positive expression.

\subsection{Statistical Analyses}

Statistical analyses were performed using the SPSS 15.0 software package (SPSS Inc., Chicago, IL, USA). The $\chi^{2}$ and Fisher's exact tests were used for comparison between categorical variables. Cox proportional hazards models were used for univariate and multivariate analyses. The hazard ratios (HR), 95\% confidence intervals (CI), and $p$ values are reported. Kaplan-Meier survival curves were also plotted. Differences between survival times were analyzed by the log-rank method. All tests were two-sided. 


\section{Results}

\subsection{Detection of Active SRC in HNSCC Tissue Specimens}

Immunohistochemical analysis of active SRC expression was performed on tissue specimens from 122 HNSCC patients. Immunostaining was successfully evaluated in $116(95 \%)$ of the 122 cases. Of these 116 tumors, 88 (76\%) exhibited positive SRC expression preferentially detected in the cytoplasm, although some cases also displayed protein enrichment at the cell membrane (Figure $1 \mathrm{~A}-\mathrm{C}$ ). Normal epithelium showed positive staining in the basal cell layer and negligible expression in the most differentiated layers (Figure 1D). In addition, immunohistochemical analysis of p-SRC (Tyr419) and total SRC was also performed and correlated with active SRC expression (not phosphorylated at Tyr530). Positive p-SRC (Tyr419) staining was mainly detected in the nucleus in 98 (88\%) of the tumors, and some cases also exhibited cytoplasmic staining (11 tumors) (Figure 1E-H). A significant positive correlation was observed between active SRC expression and nuclear p-SRC (Tyr419) (Spearman correlation coefficient $0.305, p=0.001$ ) but not cytoplasmic p-SRC (Tyr419) (Spearman correlation coefficient -0.023, $p=0.810$ ). Concordantly, total SRC expression also exhibited both nuclear and cytoplasmic patterns (Figure 1I-K) and showed a significant correlation with active SRC expression (Spearman correlation coefficient $0.318, p=0.001$ ). The expression of $\mathrm{p}$-SRC (Tyr419) was also confirmed by Western blot analysis in a subset of tumor samples compared to patient-matched normal tissues and in a panel of HNSCC-derived cell lines (Supplementary Materials Figure S1). Consistent with the IHC data, p-SRC (Tyr419) levels were increased in tumors compared to patient-matched normal epithelia (Patients 1-3) as well as HNSCC cells. Total SRC expression levels were also found to increase in tumors compared to the normal counterparts (Patients 1,2, and 4).

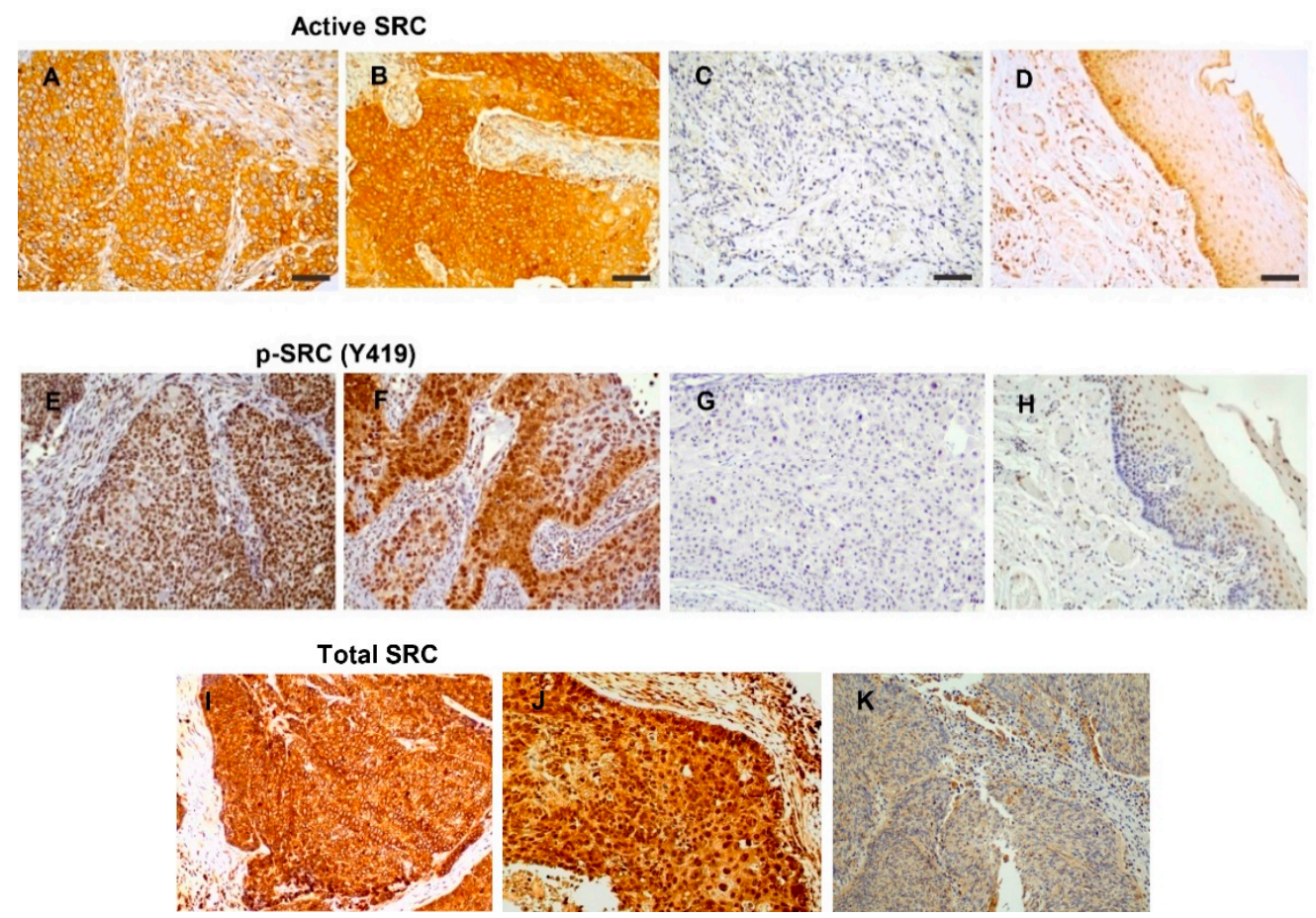

Figure 1. Immunohistochemical analysis of SRC expression in head and neck squamous cell carcinoma (HNSCC) tissue specimens. Representative examples of HNSCC showing positive active SRC staining (A,B, cytoplasmic and membrane enrichment), negative staining (C), and normal adjacent epithelia (D). Representative examples of tumors with positive p-SRC (Tyr419) staining (E, nuclear and F, cytoplasmic and nuclear), negative staining $(\mathbf{G})$, and normal adjacent epithelia $(\mathbf{H})$. Representative examples of tumors showing positive total SRC staining (I,J, cytoplasmic and nuclear) and negative staining (K). Magnification 20×. Scale bars $=50 \mu \mathrm{m}$. 


\subsection{Correlations with Clinicopathological Parameters and Disease Outcome}

We next assessed the associations between expression of active SRC and the clinicopathological parameters and disease outcome. As shown in Table 1, positive SRC expression was significantly associated with the presence of lymph node metastasis $(p=0.020)$, and advanced disease stage $(p=0.048)$. In addition, SRC expression was strongly and significantly associated with tumor recurrence $(p=0.002)$. Accordingly, patients carrying SRC-positive tumors showed a significantly higher rate of cancer-related deaths $(p=0.001)$ (Table 1$)$.

\subsection{Differential Clinical Significance of Active SRC Expression Depending on the Tumor Location}

When the clinical relevance of active SRC was analyzed separately by tumor site in our cohort of HNSCC patients, striking differences were observed (Table 2). Positive SRC expression was more frequent in bigger tumor sizes $(\mathrm{T} 3, \mathrm{~T} 4)$ and cases with lymph node metastasis $(\mathrm{N}+)$ in both locations, although the differences were not statistically significant. It is noteworthy, however, that active SRC expression was strongly and significantly correlated with tumor recurrences and tumor-associated deaths specifically in the larynx $(p<0.001)$ but not the pharynx $(p=1.00)$.

Table 2. Associations of active SRC expression with clinical and follow-up data for patients with pharyngeal and laryngeal tumors.

\begin{tabular}{|c|c|c|c|c|c|c|}
\hline \multirow{2}{*}{ Characteristic } & \multicolumn{3}{|c|}{ Pharynx $($ No. $=58)$} & \multicolumn{3}{|c|}{ Larynx $($ No. $=58)$} \\
\hline & No. & Active SRC (\%) & $P$ & No. & Active SRC (\%) & $p$ \\
\hline \multicolumn{7}{|l|}{ pT classification } \\
\hline $\mathrm{T} 1, \mathrm{~T} 2$ & 17 & $13(76)$ & & 15 & $8(53)$ & \\
\hline $\mathrm{T} 3, \mathrm{~T} 4$ & 41 & $35(85)$ & $0.458^{\dagger}$ & 43 & $32(74)$ & $0.194^{\dagger}$ \\
\hline \multicolumn{7}{|l|}{ pN classification } \\
\hline N0 & 10 & $7(70)$ & & 29 & $17(59)$ & $0.155^{\dagger}$ \\
\hline N1-3 & 48 & $41(85)$ & $0.353^{\dagger}$ & 29 & $23(79)$ & \\
\hline \multicolumn{7}{|l|}{ Disease stage } \\
\hline I, II & 5 & $4(80)$ & $1.000^{+}$ & 10 & $4(40)$ & $0.055^{\dagger}$ \\
\hline III, IV & 53 & $44(83)$ & & 48 & $36(75)$ & \\
\hline \multicolumn{7}{|l|}{ Pathological grade } \\
\hline Well differentiated & 12 & $10(83)$ & & 21 & $12(57)$ & \\
\hline Moderately differentiated & 26 & $22(85)$ & 0.917 \# & 27 & $19(70)$ & $0.177^{\#}$ \\
\hline Poorly differentiated & 20 & $16(80)$ & & 10 & $9(90)$ & \\
\hline \multicolumn{7}{|l|}{ Tumor Recurrence $\ddagger$} \\
\hline No & 8 & $7(88)$ & $1.000^{+}$ & 18 & $7(39)$ & $<0.001^{+}$ \\
\hline Yes & 42 & $34(81)$ & & 26 & $24(92)$ & \\
\hline \multicolumn{7}{|l|}{ Disease status } \\
\hline Alive without disease & 10 & $8(80)$ & $1.000^{+}$ & 20 & $8(40)$ & $<0.001^{+}$ \\
\hline Dead of index cancer & 40 & $33(83)$ & & 24 & $23(96)$ & \\
\hline Died of other causes & 8 & $7(88)$ & & 14 & $9(64)$ & \\
\hline
\end{tabular}

${ }^{\#}$ Chi-square and ${ }^{\dagger}$ Fisher's exact tests. ${ }^{\ddagger}$ The 8 patients (pharynx group) and 14 patients (larynx group) who died from causes not related to the index tumor were excluded from the recurrence analysis.

\subsection{Impact of SRC Expression on Patients' Survival}

Univariate survival analysis showed a significant correlation between SRC expression and reduced disease-specific survival in the total cohort of HNSCC patients (log-rank test, $p=0.002$; Figure 2A). Interestingly, clear differences were observed when we evaluated the impact of SRC expression on patient survival in the different tumor locations. Thus, active SRC expression was significantly correlated with a shorter disease-specific survival in the larynx (log-rank test, $p<0.001$ ) but not in the pharynx (log-rank test, $p=0.940$; Figure 2B,C). Multivariate Cox analysis including pT classification 
(dichotomized as T1,T2 versus T3,T4), pN classification (dichotomized as N0 versus N+), and active SRC expression further demonstrated that active SRC expression (HR = 12.78; 95\% CI 1.7-96.11; $p=0.013)$ and cervical lymph node metastasis (HR $=3.60 ; 95 \%$ CI 1.39-9.285; $p=0.008)$ were potent independent predictors of shorter disease-specific survival in patients with laryngeal tumors.

Total HNSCC cohort
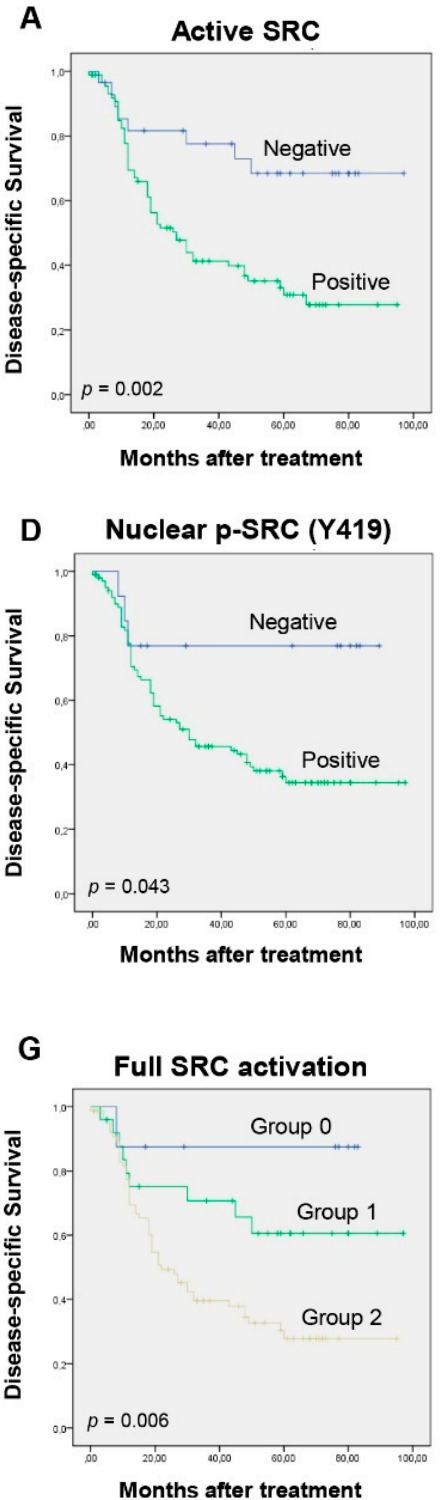

Larynx
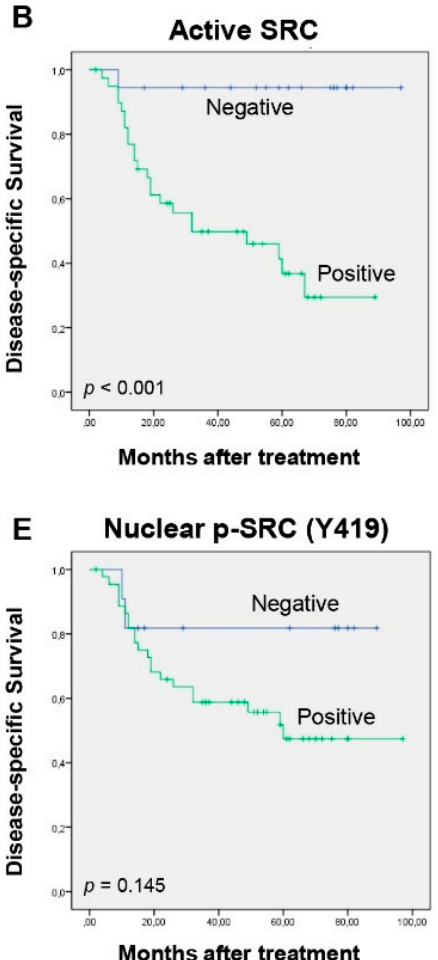

H

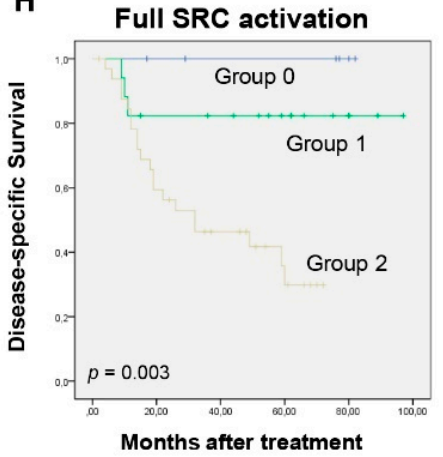

Pharynx

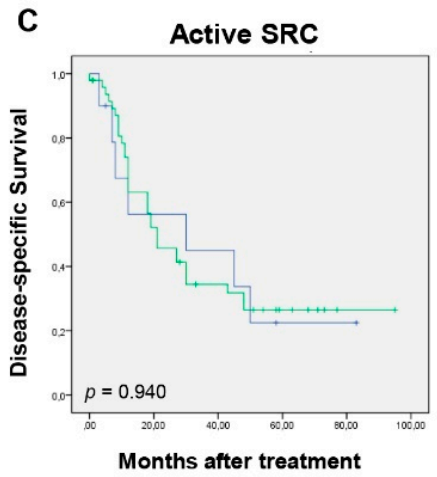

F Nuclear p-SRC (Y419)

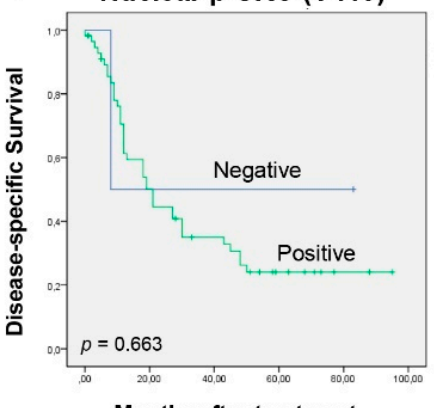

Months after treatment

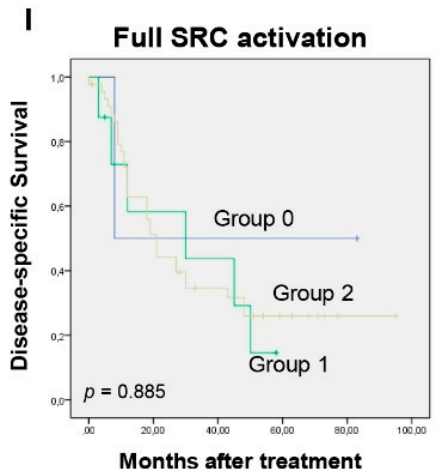

Figure 2. Impact of SRC expression on patient survival. Kaplan-Meier disease-specific survival curves categorized by active SRC expression (A-C) or nuclear p-SRC (Tyr419) (D-F) in the total cohort of HNSCC patients or in the laryngeal and pharyngeal subgroups. Kaplan-Meier disease-specific survival curves considering jointly both active SRC expression and nuclear p-SRC (Tyr419) dichotomized into three groups: positive expression of both active SRC expression and nuclear p-SRC (Tyr419) (Group 2), positive expression of either active SRC or nuclear p-SRC (Tyr419) (Group 1), or none (Group 0) (G-I) in the total HNSCC cohort and in the laryngeal and pharyngeal subgroups. $p$ values were estimated using the log-rank test.

Similarly, nuclear p-SRC (Tyr419) was also significantly associated with shorter disease-specific survival (log-rank test, $p=0.043$; Figure 2D) with marked differences depending on the tumor site (Figure 2E,F). Furthermore, the possible impact of full SRC activation on patient prognosis was assessed 
by analyzing simultaneously active SRC expression and p-SRC (Tyr419). Interestingly, we found that patients harboring both active SRC expression and p-SRC (Tyr419) (Group 2) exhibited significantly shorter survival than did patients with positive expression of either active SRC or p-SRC (Tyr419) (Group 1) or than did patients with negative expression of both active SRC expression and p-SRC (Tyr419) (Group 0) (log-rank test, $p=0.006$; Figure 2G). Furthermore, these differences were specifically observed in the larynx (log-rank test, $p=0.003$; Figure $2 \mathrm{H})$, but not the pharynx (log-rank test, $p=0.885$; Figure 2I).

\subsection{Correlations between the Expression of Active SRC and SRC-Related Proteins}

We next evaluated by immunohistochemistry the expression of various SRC effectors, such as CTTN, FAK, and ASAP1, known to regulate tumor invasion, metastasis, and aggressive phenotypes in HNSCC and other cancers [11-17,37-39]. Positive CTTN, FAK, and ASAP1 expression was respectively detected in $59(51 \%), 83(72 \%)$, and $64(55 \%)$ tumors in our cohort of HNSCC patients, predominantly cytoplasmic and with membrane enrichment in some cases (Figure 3). The expression levels of FAK and ASAP1 were found to significantly correlate with positive SRC expression $(p=0.015$ and $p=0.017$, respectively; Table 3). The potassium channel HERG1 was previously reported to form complexes with FAK [40], and is associated with aggressive tumor behavior and poor prognosis in HNSCC [41]. The expression of HERG1 was also found to significantly correlate with SRC expression in our HNSCC cohort $(p=0.011$, Table 3$)$.
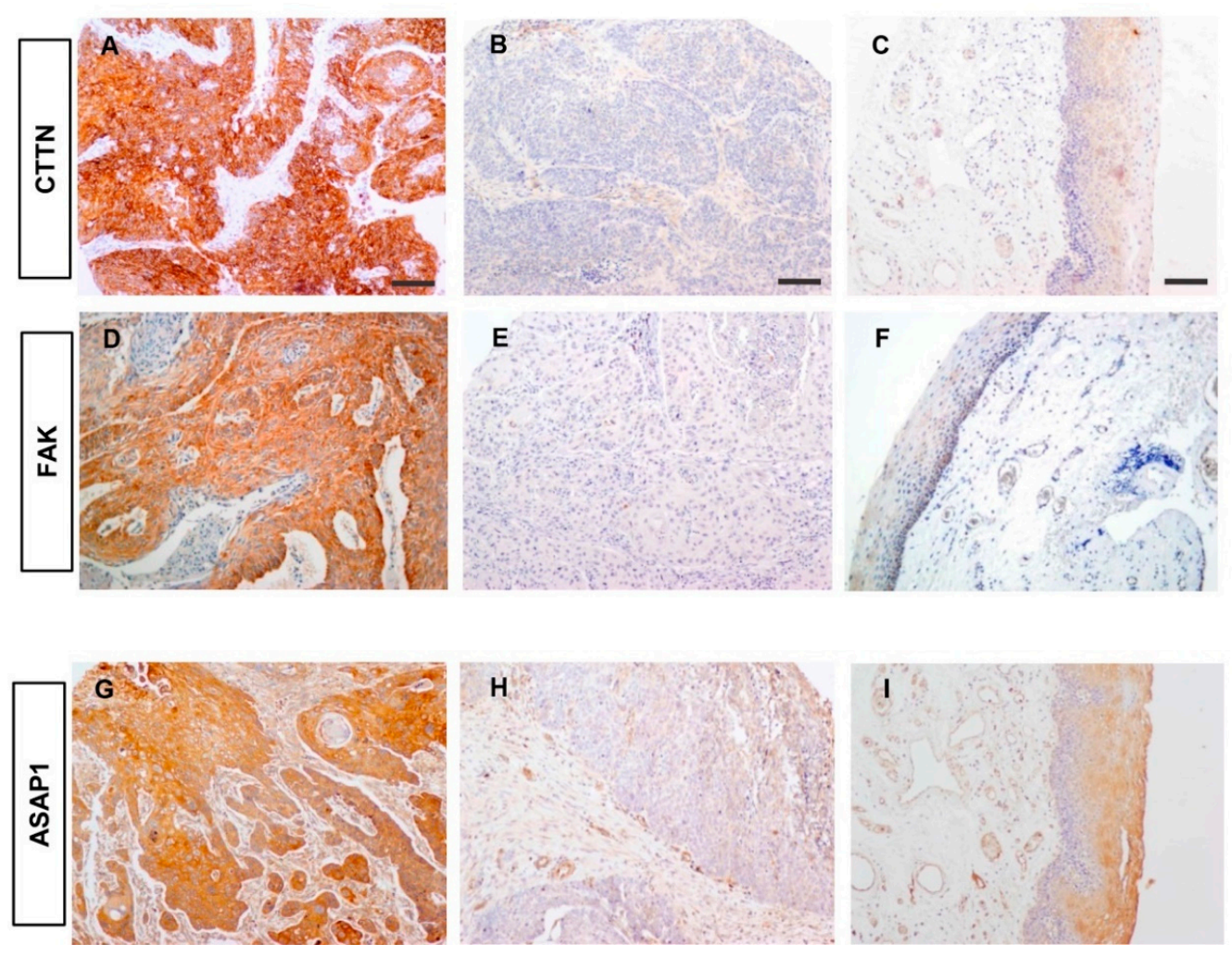

Figure 3. Immunohistochemical analysis of downstream SRC-related proteins in HNSCC tissue specimens. Representative examples of HNSCC showing strong positive cortactin (CTTN) staining (A), negative staining (B), and normal adjacent epithelia (C). Representative examples of tumors with positive focal adhesion kinase (FAK) staining (D), negative staining (E), and normal adjacent epithelia (F). Representative examples of tumors with positive staining for the Arf GTPase-activating protein ASAP1 $(\mathbf{G})$, negative staining $(\mathbf{H})$, and normal adjacent epithelia (I). Magnification 10x. Scale bars $=100 \mu \mathrm{m}$. 
Table 3. Associations between the expression of active SRC and downstream SRC-related proteins.

\begin{tabular}{cccc}
\hline Molecular Feature & No. & Active SRC (\%) & $p^{\#}$ \\
\hline CTTN protein expression & & & $(0.050)$ \\
Negative & 57 & $42(74)$ & 0.594 \\
Positive (scores 2-3) & 59 & $46(78)$ & $(0.225)$ \\
\hline FAK protein expression & & & 0.015 \\
$\quad$ Negative & 33 & $20(61)$ & $(0.221)$ \\
Positive (scores 2-3) & 83 & $68(82)$ & 0.017 \\
ASAP1 protein expression & & $34(65)$ & $(0.240)$ \\
$\quad$ Negative & 52 & $54(84)$ & 0.011 \\
Positive (scores 2-3) & 64 & $7(50)$ & $79(81)$ \\
\hline HERG1 protein expression & & 14 & \\
Negative & 98 & Positive (scores 2-3) &
\end{tabular}

When examining separately each tumor site subgroup (Table 4), we strikingly observed that the correlations between SRC expression and the SRC-related proteins FAK, ASAP1, and HERG1 were specific to the larynx and not the pharynx. Consistent with these results, the expression of the SRC substrates FAK, CTTN, and ASAP1 also showed a distinct impact on patient survival depending on the tumor site (Figure 4).

Table 4. Associations between active SRC expression and SRC-related proteins by tumor site.

\begin{tabular}{|c|c|c|c|c|c|c|}
\hline \multirow{2}{*}{ Molecular Feature } & \multicolumn{3}{|c|}{ Pharynx $($ No. $=58)$} & \multicolumn{3}{|c|}{ Larynx $($ No. $=58)$} \\
\hline & No. & Active SRC (\%) & $p^{\#}$ & No. & Active SRC (\%) & $p^{\#}$ \\
\hline $\begin{array}{l}\text { FAK protein } \\
\text { expression }\end{array}$ & & & & & & $(0.326)$ \\
\hline Negative & 14 & $11(79)$ & $(0.063)$ & 19 & $9(47)$ & \\
\hline Positive (scores 2-3) & 44 & $37(85)$ & 0.641 & 39 & $31(79)$ & 0.013 \\
\hline $\begin{array}{l}\text { ASAP1 protein } \\
\text { expression }\end{array}$ & & & & & & $(0.230)$ \\
\hline Negative & 20 & $15(75)$ & $(0.149)$ & 32 & $19(59)$ & \\
\hline Positive (scores 2-3) & 38 & $33(87)$ & 0.264 & 26 & $21(81)$ & 0.082 \\
\hline $\begin{array}{l}\text { HERG1 protein } \\
\text { expression }\end{array}$ & & & & & & $(0.366)$ \\
\hline Negative & 5 & $4(80)$ & $(0.02)$ & 9 & $3(33)$ & \\
\hline Positive (scores 2-3) & 52 & $43(83)$ & 0.882 & 46 & $36(80)$ & 0.006 \\
\hline
\end{tabular}

\# Spearman correlation coefficient (in parentheses) with the associated $p$ value. 
Total HNSCC cohort

A

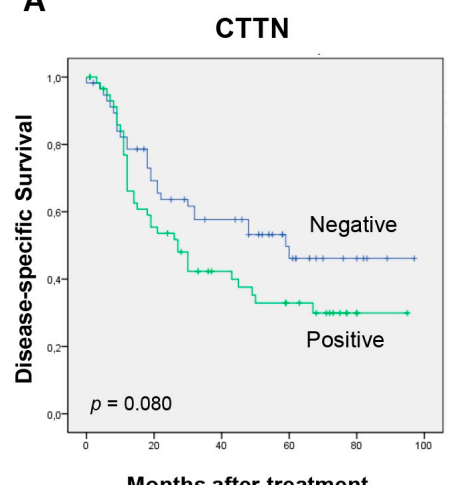

D

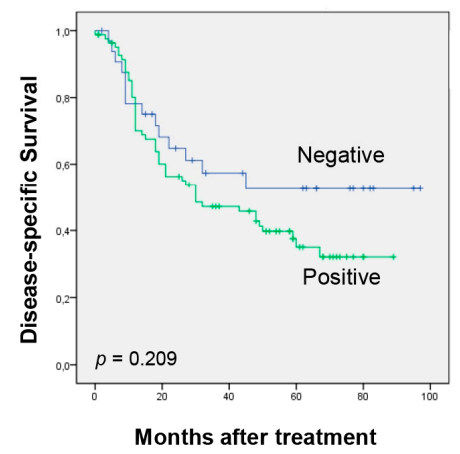

G

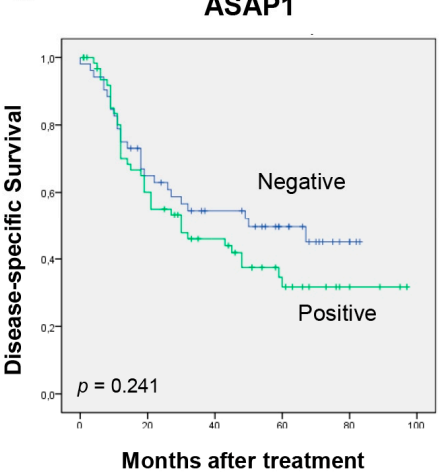

Larynx

B

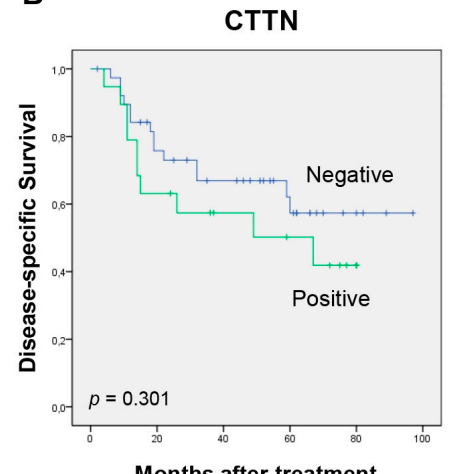

$\mathbf{E}$

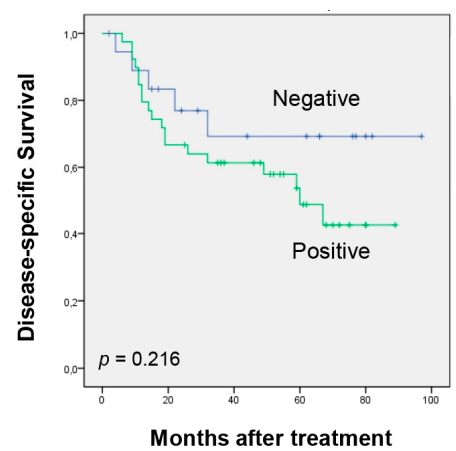

H

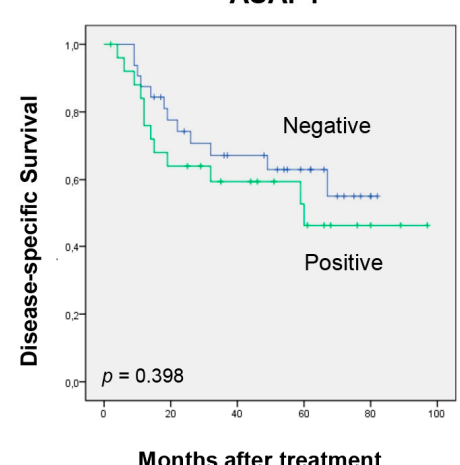

Pharynx

\section{C}

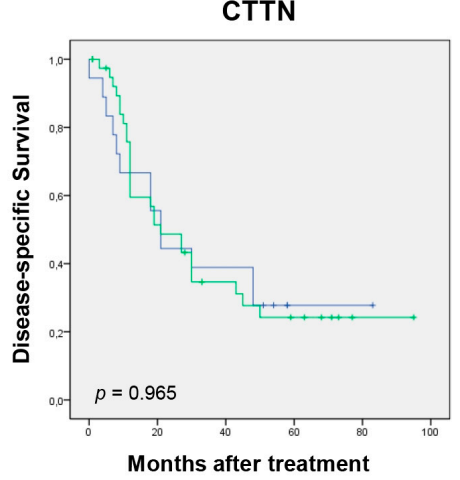

F

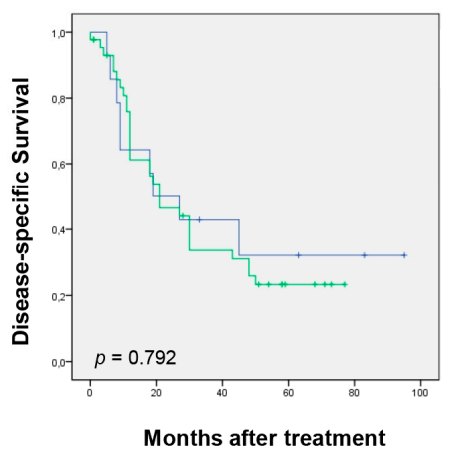

I

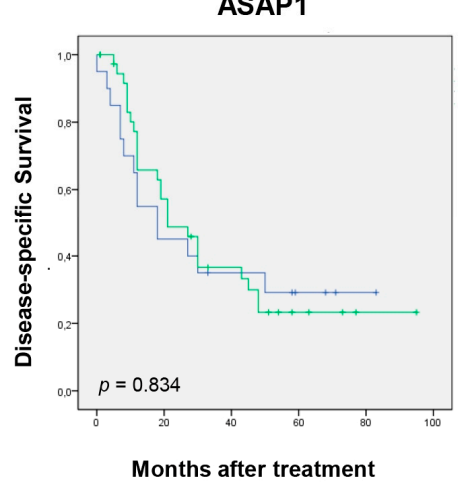

Figure 4. Impact of SRC-related proteins on patient survival. Kaplan-Meier disease-specific survival curves categorized by CTTN expression (A-C), FAK expression (D-F), and ASAP1 expression (G-I) in the total cohort of HNSCC patients or in the laryngeal and pharyngeal subgroups. $p$ values were estimated using the log-rank test.

\section{Discussion}

Aberrant SRC expression and activation is frequent in a wide variety of cancers and has been identified as a central node in numerous oncogenic pathways, thereby playing critical roles in tumor formation, progression, and dissemination [4-9]. Hence, targeting SRC has emerged as a promising therapeutic strategy for cancer treatment, and a number of SRC inhibitors have subsequently been developed and tested [4,21-30]. Overall, SRC inhibitors have demonstrated potent anti-tumor activity in preclinical models, although they are largely ineffective for the treatment of late-stage solid tumors [21,23-25,29,30]. In the specific context of HNSCC, dasatinib and saracatinib robustly inhibited cell proliferation, migration, and invasion in preclinical models [31,32,42,43]; however, these 
compounds did not show clinical efficacy as monotherapy in patients with advanced metastatic disease $[33,34]$. This therefore reflects the need for accurate response markers and adequate patient stratification to guide treatment with SRC inhibitors, which will undoubtedly contribute to improving clinical effectiveness and disease outcome.

It seems quite reasonable that those tumors harboring aberrant SRC activation and function are more likely to be effectively targeted by anti-SRC therapies. Indeed, SRC pathway activation has been positively correlated with sensitivity to treatment with dasatinib and saracatinib in different cancer types [44-46], suggesting that SRC activation could potentially serve as a biomarker to guide SRC targeting and clinical efficacy. The present study investigated the clinical significance of active SRC expression in HNSCC patients, by means of immunohistochemical detection with Clone 28 antibody specifically recognizing the active form of SRC [35,47]. In addition, the expression levels of p-SRC (Tyr419), total SRC, and various downstream SRC-related proteins were also evaluated by IHC and revealed significant correlations with active SRC expression in the total HNSCC cohort and the laryngeal subgroup. Notably, our results evidenced clear differences in the clinical impact of active SRC expression on patient survival depending on the tumor site. Active SRC specifically emerged as an independent predictor of cancer-specific mortality in patients with laryngeal tumors, but not in the pharyngeal subgroup.

Consistent with these findings, the expression of p-SRC (Tyr419) and the SRC substrates FAK and ASAP1 also showed specific associations with poor prognosis in the larynx. We also found that CTTN was another specific predictor of poor prognosis in the larynx but not in the pharynx, in agreement with our previous study [37]. However, a significant correlation between CTTN and SRC expression was not observed in our HNSCC group. Together these observations fit with the well-established oncogenic role of SRC and its downstream effectors favoring aggressive tumor phenotypes by promoting invasion and metastatic dissemination. Specifically, CTTN, FAK, and ASAP1 have been demonstrated to be key regulators of tumor invasion, metastasis, and aggressive phenotypes in HNSCC and other cancers [11-17,37-39]. In addition, we previously uncovered that the potassium channel HERG1 plays a fundamental role in early stages of HNSCC tumorigenesis and disease progression [41]. HERG1 expression was thus associated with aggressive tumor behavior and poor prognosis. This information was further and significantly extended in the present study, thereby uncovering a differential association of HERG1 expression with SRC expression depending on the HNSCC tumor site.

Similarly, SRC expression has been correlated with nodal metastasis, advanced clinical stages, recurrence, and poor prognosis in patients with oral carcinomas [48]. Increased p-SRC (Tyr419) levels have also been detected in nasopharyngeal carcinomas, both in tissue samples and in plasma, and correlated with tumor aggressiveness, distant metastasis, and unfavorable prognosis [49]. More controversially, SRC inhibitor monotherapy did not demonstrate any significant benefit in patients with metastatic HNSCC. These studies were performed in unselected cohorts of patients. Likewise, the lack of effectiveness could reflect the involvement of SRC in the first steps of the metastatic cascade to facilitate the migration and invasion of tumor cells, but not in the late stage when tumors are already disseminated.

In addition, we recently demonstrated that dasatinib and saracatinib enhanced cancer stem cell (CSC) properties in HNSCC models [50], which could also represent a plausible underlying reason to explain the lack of clinical efficacy as monotherapy in HNSCC patients. Remarkably, while dasatinib was unable to eliminate CSC subpopulations or to exhibit any significant anti-cancer activity in mouse xenografts, the mithramycin analog EC-8042 effectively targeted these deleterious effects and robustly diminished tumor growth in vivo. In line with these findings, it has been reported that dasatinib worsened the anti-neoplastic effects of cetuximab and radiation in mouse HNSCC models [51]. Moreover, saracatinib showed no effect on tumor growth but blocked perineural invasion and nodal metastasis in an orthotopic model of oral cancer [52]. Similarly, the EGFR inhibitor erlotinib, but not dasatinib, was found to significantly reduce tumor size in a randomized trial with operable 
HNSCC patients [53]. Together these data reinforce a major role for SRC favoring tumor invasion and metastasis rather than sustaining tumor growth, in good agreement with the presented results herein.

As an attempt to enhance the clinical benefit of SRC inhibitors, various combinational regimens with other anti-cancer agents have been designed and have shown promising results. Targeting SRC was effective in overcoming trastuzumab resistance and eliminating trastuzumab-resistant tumors in vivo [54]. SRC inhibition was also shown to overcome resistance to HER2 inhibitors, restoring lapatinib sensitivity [55], and to reduce tumor growth in Met-driven tumors [56]. Dual inhibition of SRC and MET led to synergistic cytotoxic effects in HNSCC models [57], and this combination targeting has also emerged as a promising therapeutic strategy for colon cancer [58]. In addition, combination treatment of dasatinib with EC-8042 in HNSCC models demonstrated favorable complementary anti-proliferative, anti-stemness, and anti-invasive effects [50], suggesting this novel combinational strategy for clinical testing in HNSCC patients. Nevertheless, it should be emphasized that adequate preselection of patients who may respond to and benefit from anti-SRC therapies is fundamental to guide clinical trials, as well as to identify reliable response biomarkers to improve stratification, treatment efficacy, and, ultimately, clinical outcome.

\section{Conclusions}

We herein provided unprecedented evidence for the differential clinical impact of SRC expression in HNSCC patients depending on the tumor site. Active SRC expression specifically emerged as an independent predictor of poor prognosis in patients with laryngeal cancer. Similarly, the expression levels of p-SRC (Tyr419) and various downstream SRC effectors such as FAK and ASAP1 significantly and consistently correlated with active SRC expression and specifically predicted poor prognosis in the larynx. Our findings could have important implications for various ongoing SFK-based clinical trials, as these new criteria could help to improve the selection and biomarker-based stratification of patients who may benefit from treatment with SRC inhibitors.

Supplementary Materials: The following are available online at http://www.mdpi.com/2072-6694/11/11/1644/s1, Figure S1: Western blot analyses of p-SRC (Tyr419) and total SRC in tumor samples and patient-matched normal mucosa from HNSCC patients, and in a panel of HNSCC-derived cell lines. Table S1: Clinical and follow-up data and IHC results from 116 HNSCC patients.

Author Contributions: Conceptualization, J.M.G.-P.; Formal analysis, R.R., J.P.R. and J.M.G.-P.; Funding acquisition, L.M.M., R.R., J.P.R. and J.M.G.-P.; Investigation, F.H.-P., R.G.-D., N.d.-R.-I., M.A.V., I.G. and C.A.-M.; Methodology, E.A. and A.V.; Resources, L.M.M. and J.P.R.; Supervision, J.M.G.-P.; Visualization, F.H.-P. and J.M.G.-P.; Writing—original draft, J.M.G.-P.; Writing—review and editing, F.H.-P., L.M.M., R.R. and J.P.R.

Funding: This study was supported by grants from the Plan Nacional de I+D+I 2013-2016 [ISCIII (PI16/00280 to JMGP and CPII16/00049 to RR), CIBERONC (CB16/12/00390 to JPR and CB16/12/00443 to LMM) and MINECO (SAF2016-75286-R to RR)], the Instituto de Investigación Sanitaria del Principado de Asturias (ISPA), Fundación Merck Salud (17-CC-008 to JPR), Ayudas a Grupos PCTI Principado de Asturias (IDI2018/155 to JPR) and the FEDER Funding Program from the European Union.

Acknowledgments: We want to particularly acknowledge for its collaboration the Principado de Asturias BioBank (PT17/0015/0023), financed jointly by Servicio de Salud del Principado de Asturias, Instituto de Salud Carlos III, and Fundación Bancaria Cajastur and integrated in the Spanish National Biobanks Network. We also thank Juan Pérez Ortega for his excellent administrative support.

Conflicts of Interest: The authors declare no conflict of interest.

\section{References}

1. Haddad, R.I.; Shin, D.M. Recent advances in head and neck cancer. N. Engl. J. Med. 2008, 359, $1143-1154$. [CrossRef] [PubMed]

2. Leemans, C.R.; Snijders, P.J.F.; Brakenhoff, R.H. The molecular landscape of head and neck cancer. Nat. Rev. Cancer 2018, 18, 269-282. [CrossRef] [PubMed]

3. Yeatman, T.J. A renaissance for SRC. Nat. Rev. Cancer 2004, 4, 470-480. [CrossRef] [PubMed]

4. Kim, L.C.; Song, L.; Haura, E.B. Src kinases as therapeutic targets for cancer. Nat. Rev. Clin. Oncol. 2009, 6, 587-595. [CrossRef] 
5. Sen, B.; Johnson, F.M. Regulation of Src Family Kinases in Human Cancers. J. Signal Transduct. 2011, 2011, 865819. [CrossRef]

6. Summy, J.M.; Gallick, G.E. Src family kinases in tumor progression and metastasis. Cancer Metastasis Rev. 2003, 22, 337-358. [CrossRef]

7. Parsons, S.J.; Parsons, J.T. Src family kinases, key regulators of signal transduction. Oncogene 2004, 23, 7906-7909. [CrossRef]

8. Frame, M.C. Src in cancer: Deregulation and consequences for cell behaviour. Biochim. Biophys. Acta 2002, 1602, 114-130. [CrossRef]

9. Irby, R.B.; Yeatman, T.J. Role of Src expression and activation in human cancer. Oncogene 2000, 19, 5636-5642. [CrossRef]

10. Ishizawar, R.; Parsons, S.J. c-Src and cooperating partners in human cancer. Cancer Cell 2004, 6, $209-214$. [CrossRef]

11. Mandal, M.; Myers, J.N.; Lippman, S.M.; Johnson, F.M.; Williams, M.D.; Rayala, S.; Ohshiro, K.; Rosenthal, D.I.; Weber, R.S.; Gallick, G.E.; et al. Epithelial to mesenchymal transition in head and neck squamous carcinoma: Association of Src activation with E-cadherin downregulation, vimentin expression, and aggressive tumor features. Cancer 2008, 112, 2088-2100. [CrossRef] [PubMed]

12. Avizienyte, E.; Frame, M.C. Src and FAK signaling controls adhesion fate and the epithelial-to-mesenchymal transition. Curr. Opin. Cell Biol. 2005, 17, 542-547. [CrossRef] [PubMed]

13. Schlaepfer, D.D.; Jones, K.C.; Hunter, T. Multiple Grb2-mediated integrin-stimulated signaling pathways to ERK2/mitogen-activated protein kinase: Summation of both c-Src and focal adhesion kinase initiated tyrosine phosphorylation events. Mol. Cell Biol. 1998, 18, 2571-2585. [CrossRef] [PubMed]

14. Chang, J.H.; Gill, S.; Settleman, J.; Parsons, S.J. c-Src regulates the simultaneous rearrangement of actin cytoskeleton, p190RhoGAP, and p120RasGAP following epidermal growth factor stimulation. J. Cell Biol. 1995, 130, 355-368. [CrossRef] [PubMed]

15. Wang, W.; Liu, Y.; Liao, K. Tyrosine phosphorylation of cortactin by the FAK-Src complex at focal adhesions regulates cell motility. BMC Cell Biol. 2011, 12, 49. [CrossRef] [PubMed]

16. Onodera, Y.; Hashimoto, S.; Hashimoto, A.; Morishige, M.; Mazaki, Y.; Yamada, A.; Ogawa, E.; Adachi, M.; Sakurai, T.; Manabe, T.; et al. Expression of AMAP1, an ArfGAP, provides novel targets to inhibit breast cancer invasive activities. EMBO J. 2005, 24, 963-973. [CrossRef]

17. Hashimoto, S.; Furukawa, S.; Hashimoto, A.; Tsutaho, A.; Fukao, A.; Sakamura, Y.; Parajuli, G.; Onodera, Y.; Otsuka, Y.; Handa, H.; et al. ARF6 and AMAP1 are major targets of KRAS and TP53 mutations to promote invasion, PD-L1 dynamics, and immune evasion of pancreatic cancer. Proc. Natl Acad. Sci. USA 2019, 116, 17450-17459. [CrossRef]

18. Masaki, T.; Igarashi, K.; Tokuda, M.; Yukimasa, S.; Han, F.; Jin, Y.J.; Li, J.Q.; Yoneyama, H.; Uchida, N.; Fujita, J.; et al. pp60c-src activation in lung adenocarcinoma. Eur. J. Cancer 2003, 39, 1447-1455. [CrossRef]

19. Talamonti, M.S.; Roh, M.S.; Curley, S.A.; Gallick, G.E. Increase in activity and level of pp60c-src in progressive stages of human colorectal cancer. J. Clin. Invest. 1993, 91, 53-60. [CrossRef]

20. Alvarez, R.H.; Kantarjian, H.M.; Cortes, J.E. The role of Src in solid and hematologic malignancies: Development of new-generation Src inhibitors. Cancer 2006, 107, 1918-1929. [CrossRef]

21. Zhang, S.; Yu, D. Targeting Src family kinases in anti-cancer therapies: Turning promise into triumph. Trends Pharmacol. Sci. 2012, 33, 122-128. [CrossRef] [PubMed]

22. Elsberger, B.; Stewart, B.; Tatarov, O.; Edwards, J. Is Src a viable target for treating solid tumours? Curr. Cancer Drug Targets 2010, 10, 683-694. [CrossRef] [PubMed]

23. Creedon, H.; Brunton, V.G. Src kinase inhibitors: Promising cancer therapeutics? Crit. Rev. Oncog. 2012, 17, 145-159. [CrossRef] [PubMed]

24. Schuetze, S.M.; Bolejack, V.; Choy, E.; Ganjoo, K.N.; Staddon, A.P.; Chow, W.A.; Tawbi, H.A.; Samuels, B.L.; Patel, S.R.; von Mehren, M.; et al. Phase 2 study of dasatinib in patients with alveolar soft part sarcoma, chondrosarcoma, chordoma, epithelioid sarcoma, or solitary fibrous tumor. Cancer 2017, 123, 90-97. [CrossRef] [PubMed]

25. Scott, A.J.; Song, E.K.; Bagby, S.; Purkey, A.; McCarter, M.; Gajdos, C.; Quackenbush, K.S.; Cross, B.; Pitts, T.M.; Tan, A.C.; et al. Evaluation of the efficacy of dasatinib, a Src/Abl inhibitor, in colorectal cancer cell lines and explant mouse model. PLoS ONE 2017, 12, e0187173. [CrossRef] [PubMed] 
26. Gore, L.; Kearns, P.R.; de Martino, M.L.; Lee; De Souza, C.A.; Bertrand, Y.; Hijiya, N.; Stork, L.C.; Chung, N.G.; Cardos, R.C.; et al. Dasatinib in Pediatric Patients With Chronic Myeloid Leukemia in Chronic Phase: Results From a Phase II Trial. J. Clin. Oncol. 2018, 36, 1330-1338. [CrossRef]

27. Ocana, A.; Gil-Martin, M.; Martín, M.; Rojo, F.; Antolín, S.; Guerrero, Á.; Trigo, J.M.; Muñoz, M.; Pandiella, A.; Diego, N.G.; et al. A phase I study of the SRC kinase inhibitor dasatinib with trastuzumab and paclitaxel as first line therapy for patients with HER2-overexpressing advanced breast cancer. GEICAM/2010-04 study. Oncotarget 2017, 8, 73144-73153. [CrossRef]

28. Montemurro, M.; Cioffi, A.; Dômont, J.; Rutkowski, P.; Roth, A.D.; von Moos, R.; Inauen, R.; Toulmonde, M.; Burkhard, R.O.; Knuesli, C.; et al. Long-term outcome of dasatinib first-line treatment in gastrointestinal stromal tumor: A multicenter, 2-stage phase 2 trial (Swiss Group for Clinical Cancer Research 56/07). Cancer 2018, 124, 1449-1454. [CrossRef]

29. Kalinsky, K.; Lee, S.; Rubin, K.M.; Lawrence, D.P.; Iafrarte, A.J.; Borger, D.R.; Margolin, K.A.; Leitao, M.M., Jr.; Tarhini, A.A.; Koon, H.B.; et al. A phase 2 trial of dasatinib in patients with locally advanced or stage IV mucosal, acral, or vulvovaginal melanoma: A trial of the ECOG-ACRIN Cancer Research Group (E2607). Cancer 2017, 123, 2688-2697. [CrossRef]

30. Parseghian, C.M.; Parikh, N.U.; Wu, J.Y.; Jiang, Z.Q.; Henderson, L.; Tian, F.; Pastor, B.; Ychou, M.; Raghav, K.; Dasari, A.; et al. Dual Inhibition of EGFR and c-Src by Cetuximab and Dasatinib Combined with FOLFOX Chemotherapy in Patients with Metastatic Colorectal Cancer. Clin. Cancer Res. 2017, 23, 4146-4154. [CrossRef]

31. Johnson, F.M.; Saigal, B.; Talpaz, M.; Donato, N.J. Dasatinib (BMS-354825) tyrosine kinase inhibitor suppresses invasion and induces cell cycle arrest and apoptosis of head and neck squamous cell carcinoma and non-small cell lung cancer cells. Clin. Cancer Res. 2005, 11, 6924-6932. [CrossRef] [PubMed]

32. Green, T.P.; Fennell, M.; Whittaker, R.; Curwen, J.; Jacobs, V.; Allen, J.; Logie, A.; Hargreaves, J.; Hickinson, D.M.; Wilkinson, R.W.; et al. Preclinical anticancer activity of the potent, oral Src inhibitor AZD0530. Mol. Oncol. 2009, 3, 248-261. [CrossRef] [PubMed]

33. Brooks, H.D.; Glisson, B.S.; Bekele, B.N.; Johnson, F.M.; Ginsberg, L.E.; El-Naggar, A.; Culotta, K.S.; Takebe, N.; Wright, J.; Tran, H.T.; et al. Phase 2 study of dasatinib in the treatment of head and neck squamous cell carcinoma. Cancer 2011, 117, 2112-2119. [CrossRef] [PubMed]

34. Fury, M.G.; Baxi, S.; Shen, R.; Kelly, K.W.; Lipson, B.L.; Carlson, D.; Stambuk, H.; Haque, S.; Pfister, D.G. Phase II study of saracatinib (AZD0530) for patients with recurrent or metastatic head and neck squamous cell carcinoma (HNSCC). Anticancer Res. 2011, 31, 249-253.

35. Kawakatsu, H.; Sakai, T.; Takagaki, Y.; Shinoda, Y.; Saito, M.; Owada, M.K.; Yano, J. A new monoclonal antibody which selectively recognizes the active form of Src tyrosine kinase. J. Biol. Chem. 1996, 271, 5680-5685. [CrossRef]

36. Rodrigo, J.P.; Hermsen, M.A.; Fresno, M.F.; Brakenhoff, R.H.; García-Velasco, F.; Snijders, P.J.; Heideman, D.A.; García-Pedrero, J.M. Prevalence of human papillomavirus in laryngeal and hypopharyngeal squamous cell carcinomas in northern Spain. Cancer Epidemiol. 2015, 39, 37-41. [CrossRef]

37. Rodrigo, J.P.; García-Carracedo, D.; García, L.A.; Menéndez, S.; Allonca, E.; González, M.V.; Fresno, M.F.; Suárez, C.; García-Pedrero, J.M. Distinctive clinicopathological associations of amplification of the cortactin gene at 11q13 in head and neck squamous cell carcinomas. J. Pathol. 2009, 217, 516-523. [CrossRef]

38. Canel, M.; Secades, P.; Garzón-Arango, M.; Allonca, E.; Suarez, C.; Serrels, A.; Frame, M.; Brunton, V.; Chiara, M.D. Involvement of focal adhesion kinase in cellular invasion of head and neck squamous cell carcinomas via regulation of MMP-2 expression. Br. J. Cancer 2008, 98, 1274-1284. [CrossRef]

39. Sato, H.; Hatanaka, K.C.; Hatanaka, Y.; Hatakeyama, H.; Hashimoto, A.; Matsuno, Y.; Fukuda, S.; Sabe, H. High level expression of AMAP1 protein correlates with poor prognosis and survival after surgery of head and neck squamous cell carcinoma patients. Cell Commun. Signal 2014, 12, 17. [CrossRef]

40. Becchetti, A.; Crescioli, S.; Zanieri, F.; Petroni, G.; Mercatelli, R.; Coppola, S.; Gasparoli, L.; D'Amico, M.; Pillozzi, S.; Crociani, O.; et al. The conformational state of hERG1 channels determines integrin association, downstream signaling, and cancer progression. Sci. Signal 2017, 10, eaaf3236. [CrossRef]

41. Menéndez, S.T.; Rodrigo, J.P.; Alvarez-Teijeiro, S.; Villaronga, M.Á.; Allonca, E.; Vallina, A.; Astudillo, A.; Barros, F.; Suárez, C.; García-Pedrero, J.M. Role of HERG1 potassium channel in both malignant transformation and disease progression in head and neck carcinomas. Mod. Pathol. 2012, 25, 1069-1078. [CrossRef] [PubMed] 
42. Veracini, L.; Grall, D.; Schaub, S.; Beghelli-de la Forest Divonne, S.; Etienne-Grimaldi, M.C.; Milano, G.; Bozec, A.; Babin, E.; Sudaka, A.; Thariat, J.; et al. Elevated Src family kinase activity stabilizes E-cadherin-based junctions and collective movement of head and neck squamous cell carcinomas. Oncotarget 2015, 6, 7570-7583. [CrossRef] [PubMed]

43. Koppikar, P.; Choi, S.H.; Egloff, A.M.; Cai, Q.; Suzuki, S.; Freilino, M.; Nozawa, H.; Thomas, S.M.; Gooding, W.E.; Siegfried, J.M.; et al. Combined inhibition of c-Src and epidermal growth factor receptor abrogates growth and invasion of head and neck squamous cell carcinoma. Clin. Cancer Res. 2008, 14, 4284-4291. [CrossRef] [PubMed]

44. Arcaroli, J.J.; Touban, B.M.; Tan, A.C.; Varella-Garcia, M.; Powell, R.W.; Eckhardt, S.G.; Elvin, P.; Gao, D.; Messersmith, W.A. Gene array and fluorescence in situ hybridization biomarkers of activity of saracatinib (AZD0530), a Src inhibitor, in a preclinical model of colorectal cancer. Clin. Cancer Res. 2010, 16, 4165-4177. [CrossRef]

45. Nagaraj, N.S.; Smith, J.J.; Revetta, F.; Washington, M.K.; Merchant, N.B. Targeted inhibition of SRC kinase signaling attenuates pancreatic tumorigenesis. Mol. Cancer Ther. 2010, 9, 2322-2332. [CrossRef]

46. Suwaki, N.; Vanhecke, E.; Atkins, K.M.; Graf, M.; Swabey, K.; Huang, P.; Schraml, P.; Moch, H.; Cassidy, A.M.; Brewer, D.; et al. A HIF-regulated VHL-PTP1B-Src signaling axis identifies a therapeutic target in renal cell carcinoma. Sci. Transl. Med. 2011, 3, 85ra47. [CrossRef]

47. Zou, D.; Yoon, H.S.; Anjomshoaa, A.; Perez, D.; Fukuzawa, R.; Guilford, P.; Humar, B. Increased levels of active c-Src distinguish invasive from in situ lobular lesions. Breast Cancer Res. 2009, 11, R45. [CrossRef]

48. Cheng, S.J.; Kok, S.H.; Lee, J.J.; Yen-Ping Kuo, M.; Cheng, S.L.; Huang, Y.L.; Chen, H.M.; Chang, H.H.; Chiang, C.P. Significant association of SRC protein expression with the progression, recurrence, and prognosis of oral squamous cell carcinoma in Taiwan. Head Neck 2012, 34, 1340-1345. [CrossRef]

49. Ke, L.; Xiang, Y.; Guo, X.; Lu, J.; Xia, W.; Yu, Y.; Peng, Y.; Wang, L.; Wang, G.; Ye, Y.; et al. c-Src activation promotes nasopharyngeal carcinoma metastasis by inducing the epithelial-mesenchymal transition via PI3K/Akt signaling pathway: A new and promising target for NPC. Oncotarget 2016, 7, 28340-28355. [CrossRef]

50. Hermida-Prado, F.; Villaronga, M.A.; Granda-Díaz, R.; del-Río-Ibisate, N.; Santos, L.; Hermosilla, M.A.; Oro, P.; Allonca, E.; Agorreta, J.; Garmendia, I.; et al. The SRC Inhibitor Dasatinib Induces Stem Cell-Like Properties in Head and Neck Cancer Cells that are Effectively Counteracted by the Mithralog EC-8042. J. Clin. Med. 2019, 8, 1157. [CrossRef]

51. Baro, M.; de Llobet, L.I.; Figueras, A.; Skvortsova, I.; Mesia, R.; Balart, J. Dasatinib worsens the effect of cetuximab in combination with fractionated radiotherapy in FaDu- and A431-derived xenografted tumours. Br. J. Cancer 2014, 111, 1310-1318. [CrossRef] [PubMed]

52. Ammer, A.G.; Kelley, L.C.; Hayes, K.E.; Evans, J.V.; Lopez-Skinner, L.A.; Martin, K.H.; Frederick, B.; Rothschild, B.L.; Raben, D.; Elvin, P.; et al. Saracatinib Impairs Head and Neck Squamous Cell Carcinoma Invasion by Disrupting Invadopodia Function. J. Cancer Sci. Ther. 2009, 1, 52-61. [CrossRef] [PubMed]

53. Bauman, J.E.; Duvvuri, U.; Gooding, W.E.; Rath, T.J.; Gross, N.D.; Song, J.; Jimeno, A.; Yarbrough, W.G.; Johnson, F.M.; Wang, L.; et al. Randomized, placebo-controlled window trial of EGFR, Src, or combined blockade in head and neck cancer. JCI Insight 2017, 2, e90449. [CrossRef] [PubMed]

54. Zhang, S.; Huang, W.C.; Li, P.; Guo, H.; Poh, S.B.; Brady, S.W.; Xiong, Y.; Tseng, L.M.; Li, S.H.; Ding, Z.; et al. Combating trastuzumab resistance by targeting SRC, a common nodedownstream of multiple resistance pathways. Nat. Med. 2011, 17, 461-469. [CrossRef] [PubMed]

55. Rexer, B.N.; Ham, A.J.; Rinehart, C.; Hill, S.; Granja-Ingram Nde, M.; González-Angulo, A.M.; Mills, G.B.; Dave, B.; Chang, J.C.; Liebler, D.C.; et al. Phosphoproteomic mass spectrometry profiling links Src family kinases to escape from HER2 tyrosine kinase inhibition. Oncogene 2011, 30, 4163-4174. [CrossRef]

56. Bertotti, A.; Bracco, C.; Girolami, F.; Torti, D.; Gastaldi, S.; Galimi, F.; Medico, E.; Elvin, P.; Comoglio, P.M.; Trusolino, L. Inhibition of Src impairs the growth of met-addicted gastric tumors. Clin. Cancer Res. 2010, 16, 3933-3943. [CrossRef] 
57. Sen, B.; Peng, S.; Saigal, B.; Williams, M.D.; Johnson, F.M. Distinct interactions between c-Src and c-Met in mediating resistance to c-Src inhibition in head and neck cancer. Clin. Cancer Res. 2011, 17, 514-524. [CrossRef]

58. Song, N.; Qu, X.; Liu, S.; Zhang, S.; Liu, J.; Qu, J.; Zheng, H.; Liu, Y.; Che, X. Dual inhibition of MET and SRC kinase activity as a combined targeting strategy for colon cancer. Exp. Ther. Med. 2017, 14, 1357-1366. [CrossRef]

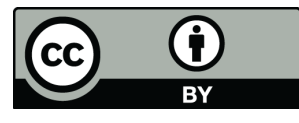

(C) 2019 by the authors. Licensee MDPI, Basel, Switzerland. This article is an open access article distributed under the terms and conditions of the Creative Commons Attribution (CC BY) license (http://creativecommons.org/licenses/by/4.0/). 